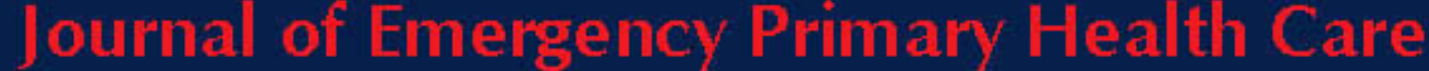

An International elournal of Prehospital care Research, Education, clinical Practice, Policy and Service Delivery

\title{
EDUCATION
}

\section{Paramedic Practice - Knowledge Invested in Action}

Article No. 990057

\section{Andrea Wyatt}

\section{Keywords}

tacit knowledge; clinical judgment; paramedic practice

\begin{abstract}
The ability of expert practitioners to make sound judgments when faced with non-routine situations calls on a form of tacit knowing that has been loosely branded as 'intuition', a 'sixth sense', or 'gut feeling'. The development of tacit knowledge is associated with nonformal learning that occurs in the context of the workplace; however, the elusive nature of this phenomenon has served to hamper research efforts. The focus has therefore shifted away from tacit knowledge toward the more observable concept of 'judgment'. Paramedics are called to make clinical judgments as part of their everyday practice, often unaware of the basis behind these judgments. This case study examines the source of knowledge drawn on by experienced paramedics when making judgments, especially when faced with situations they have not previously encountered, and proposes how new knowledge and meaning is constructed through such involvements. A better understanding of the relationship between knowing and judgment, and how they are developed, will have future implications for the provision of ongoing education and training programs for paramedics.
\end{abstract}

\section{Introduction}

The doctor has a 'hunch' that a patient's signs and symptoms are more than they seem - but he is unable to say why. The teacher 'just knows' that a student is capable of more than their assessments reveal. Expert practitioners, in making judgments and decisions as part of their professional role, often call on a particular form of knowing that is difficult to quantify and identify. It can be loosely termed 'intuition', 'gut feeling', or a 'sixth sense'. However, these terms are not useful in helping to uncover the elusiveness of this tacit form of knowing. Similarly, referring to the phenomenon as 'tacit knowledge' removes the importance of the action from the process, that is, it ignores the relationship between the 'knowing' and the 'doing'. The responsibility of educators therefore is to uncover the relationships that exist between professional's learning, the development of their knowledge, and the utilization of that knowledge in the performance of their practice.

The need to explore and better understand tacit knowledge arises through the observation of practitioners as they undergo transition from novice to expert. The experienced professional possesses a 'knowing' that calls into question the relationships between theory and experience, education and practice, skill and knowledge. The traditional epistemology of theoretical knowledge is challenged as the likes of 'experience' and 'practice' are recognised 
as equally legitimate sources of knowledge. The fundamental basis of what constitutes 'valid' knowledge is also being called into question, as '...knowledge is held to be partial and contingent upon the specific factors and contexts within which it is constructed and presented'[1]. Prevailing concepts of fundamental knowledge currently offered by formal educational institutions are being challenged as various forms of context specific knowledge and learning gain increasing recognition. The workplace is being considered a genuine site of non-formal learning and knowledge acquisition, where the ability of the expert practitioner to provide sound decision-making when confronted with unique situations is both developed and refined through a form of knowing closely aligned with experiential and practical knowledge.

In the pre-hospital context, paramedics are frequently confronted with unique situations that call for the making of almost instantaneous judgments concerning appropriate patient management strategies. For novices within this environment such judgments are often rigid and uncompromising being largely driven by established protocols. However, as the paramedic develops an experiential learning base a degree of fluency can be seen to emerge in their patient management with judgments extending beyond the boundaries of standard protocols. Such behaviour has been labeled as 'professional judgment', 'critical thinking' or simply 'intuition’ and has been closely aligned with the notion of tacit knowledge.

Attempts to isolate the knowing that is tacit from the doing that is manifest as judgment have proven to be problematic. It would appear to be more beneficial to acknowledge the combination of both theory and practice in the exercise of judgment, which Dunne[2] refers to as 'knowledge invested in action'. Similarly, Hager[3] argues that '....a theory of informal workplace learning based on practical judgment is superior to the available alternatives'. The issue is addressed by Hackley[4] who surmises that satisfactorily addressing the issue of tacit knowledge in professional judgment requires an epistemological stance that draws connections between theory and practice.

In light of the above discussions, this study was conducted to examine the utilization of tacit knowledge by experienced paramedics made explicit through their application of professional judgment.

\section{Method}

Considering the importance of operationalising the major constructs of the investigation, workplace learning and professional judgment, it followed that the design of the study should focus on ethnographic techniques that retained the contextual influences inherent in the workplace environment. The theory is embedded in the social context, and such an approach enables the collection of thick, rich descriptions that are sensitive to the socio-cultural patterns of interaction within the social order being investigated. The research method employed in this investigation was that of an ethnographic case study utilising the data collection techniques of participant observation and interview. Participant observation was selected as a research technique as it preserves the contextual influences and allows close observation of behaviour within that context. In conjunction with the data obtained from participant observation, interviews were introduced to assist the paramedics to reflect more deeply on the judgments they make in practice and make explicit the tacit knowledge on which such judgments are based. Similar studies investigating tacit knowledge or judgment within work practices have identified both participant observation and interviews as techniques suited to uncovering thick, rich data despite the elusive nature of the phenomena $[5,6,7]$. 
The phenomenon investigated in this study is the professional judgment exercised by experienced paramedics when faced with unique clinical situations and the relationship of such judgment to tacit knowledge. Therefore it was necessary for the sample group to be both senior and clinically expert paramedics in order to obtain meaningful data that would allow understanding and gain insight into such phenomenon. It follows that the most appropriate sampling strategy is non-probabilistic, or purposeful.[8] Further to this, a unique variant of purposeful sampling was adopted, with paramedics being selected because they were deemed to be exceptional operational clinicians. This would best provide data that was information rich and relevant to the research question. The above criteria were satisfied through the selection of Mobile Intensive Care (MICA) paramedics with at least four years experience who were currently acting as clinical educators. From this cohort, a final sample size of five was considered suitable, however this dropped to three following two late withdrawals. Concerns regarding the generalisability of the results obtained using the above sampling strategy, and the sample size, are of diminished relevance in this case study as it is examining relationships within an isolated context. It is recognised that this case study represents a selected part of reality, a limited view of the world. It is the responsibility of readers of the study to interpret the meaning of these results in their own context.

Approval for the study was granted by the University of Melbourne Ethics Committee and permission to observe MICA Paramedics within their work environment was obtained from the Chief Executive Officer of the Metropolitan Ambulance Service.

\section{Analysis}

Data collected from the interactions with the final three participants in the case study consisted of field notes obtained during participant observation and typed transcripts from interviews. In this study, the characteristics of tacit knowledge and judgments, as identified in a review of the literature, were adopted as major themes and formed the basis of data analysis. The validity of the research in this study was enhanced by using multiple sources (several different participants) and multiple methods (observation and interview) of data collection, confirmation of the researcher's interpretation of data with participants, and clearly defining the constructs within which the research was undertaken.

\section{Results}

\section{Role of Experience}

All participants strongly supported their experience as being a major contributor to their knowledge base and their ability to make sound clinical decisions. However the manner in which such experience was seen to be a factor in making sound judgments varied, both between participants and for individual participants. All participants saw their previous experiences as a source of stored knowledge available to assist in future decisions they made at work. This is consistent with existing theories of workplace learning, that identify the relationship between the learning experiences gained at work and the making of appropriate judgments in the workplace.[6,5] However, it was suggested that experience in isolation is not the answer to developing expertise. It is available to be drawn on, and this is indeed valuable, however it was suggested there are other factors involved.

Experience was also drawn on as a reflective tool, where it was used to guide judgments and provide an avenue for recognition of difference as well as similarities. In particular, cases that appeared routine were managed efficiently, with the paramedics seeming to know the management course and various judgment outcomes with barely a word spoken. This demonstrated an understanding of what was required and a knowledge of the standard management of such patients. Less routine cases often called for a more openly combined 
effort in the management of the patient. The paramedics discussed issues and were open to sharing their experiences in an effort to arrive at a suitable course of action.

The acquisition of experiential knowledge was seen to be broad based, with participants acknowledging diverse sources of such knowledge. There was recognition of the importance of life experiences external to the workplace environment. This is an interesting point, as much discussion has focussed around the importance of situated learning within a specific context. While not dismissing the relevance of context, several of the participant's reflections lend themselves to recognition of broader social and cultural associations, an individual's full array of life experiences.

The participants also recognised the importance of observing other paramedics operating within the workplace context and also gaining broader information from patients and their relatives.

I find my interaction with the patient I learn from, the interaction my partner has with the patient, and also when my partner is working in the patient care role, absorbing all that, absorbing information from the relatives, so there is a great deal of knowledge gained from all that. (Excerpt from interview)

The above statement is supportive of the notion of a community of practice where practitioners develop expertise through legitimate participation within the cultural and social boundaries of that community, through both direct practice and observation of experts.[9]

Despite all participants' ability to recognise experience as an important contributor toward their capacity to make sound judgments in the workplace, they also expressed an inability to adequately capture the nature of the knowledge gained, highlighting again the tacit nature of expert judgments and the difficulty in making these processes explicit.

The above discussions suggest experience was an essential element in the capacity to make sound clinical judgments in the workplace. Participants resourced this information either directly or through reflection, and obtained their experiential knowledge through various sources - life experiences, direct experience, observation of other paramedics at practice, patients and relatives of patients, other health professionals. However, despite being aware of having acquired this knowledge, there still remained some uncertainty regarding how it was accessed and made available when required in practice. It was also identified that this was not the only factor involved in the development of expertise.

\section{Learning Within a Specific Context}

It seemed more difficult for the participants to specifically relate their ability to make sound clinical judgments with the learning they had undertaken and the knowledge they had developed within the context of their workplace. Despite this, there was also a willingness to accept that making judgments was dependent on generalised or explicit knowledge as well as the context-specific knowledge obtained through actually working within a practice. This suggests that paramedics recognise the importance of gaining knowledge within the specific context of their workplace, but also recognise the importance of a strong general knowledge base that is perhaps more explicit. Certainly the literature supports the contribution of a broad range of knowledge sources,[10] both tacit and explicit; however, attention is drawn to the association between the development of tacit knowledge and expert practice. During discussions with the paramedics, they recalled their transition from ambulance paramedic to intensive care paramedic. This almost immeasurable shift in context was sufficient to nullify years of experience and relegate them to novice status - they recall adhering rigidly to 
protocols and struggling to make difficult judgments. They made similar observations of experienced nurses transferring to paramedic practice. These observations suggest knowledge developed in a specific context shapes our capacity to make judgments within that context only; however, we are often not aware of this influence until our contextual cocoon is disturbed.

\section{The Expert Paramedic's Clinical Approach}

It was interesting to discover that all participants were able to recognise the difference in thought processes, the way information is managed, between the novice and expert practitioner. This ranged from simply an identification of differences in their individual practice as they developed as practitioners to a notable distinction between the novice and the expert. One participant identifies how his clinical approach to problems has changed and is less driven by what is textbook procedure:

I suppose the clinical assessment forms a very important part of deciding what I do but there seems to be a lot of things that are very hard to explain that also fit in that picture. I remember that I had to decide to actually treat before I could get further history so my clinical assessment wasn't complete when I actually started to treat. So why did I decide to do that? And that's always a very hard question to answer. When we talk about tradition when we do that clinical assessment it is amazing how often, as you see more and more patients, you sometimes don't completely finish that clinical assessment which actually you could argue you aren't doing the right thing by the book, but there are times when you step outside that. (Excerpt from interview)

Other participants support this view:

I didn't go through all the steps, I went fairly quickly to what's the main problem. I think that's what happens more often the longer you're in the job, your senses zero in to the main problem more quickly. (excerpt from interview)

This suggests that experienced paramedics are able to identify the important clinical issues more quickly and focus on these. This is in contrast to the novice practitioner, as identified by the participants.

I think the novice would really go through the step by step approach and would really want a very clear picture of say the pain description and the whole DOLOR approach, really have a clinical assessment completely completed before they did any treatment, so I suppose in just going back a bit that basically you start doing a few things concurrentlywe actually start doing a clinical assessment whilst starting treatment for certain things while you're still exploring things further. Whereas I think a novice would really want to get each step completed before they move on to the next, they wouldn't be happy to do things concurrently. (Excerpt from interview)

There was a noticeable difference in the demeanour and the behaviour of the paramedics in several of the cases attended. At times there appeared to be no haste to initiate management of the patient, even though they were considerably unwell. On separate occasions the paramedic commenced management almost immediately without thorough examination of the patient beforehand. This behaviour would seem to indicate a certain attentiveness to the issues at hand and an ability to make judgments based on very little immediate information.

The expert practitioner's approach in analysing various clinical problems also appears to display distinct characteristics. The following statement reflects a reluctance of the expert practitioner to label patients with a specific diagnosis, but rather look at the problem in a more holistic sense. 
Well, a correct diagnosis in that setting is neither here nor there. A specific diagnosis - I believe you need to have a general direction with your management but as far as specifics, I think I was told when I first started with my first clinical instructor, he said that it would be fraught with danger if you look at a person with abdominal pain and say this appendicitis or this is acute this or that, rather than just say this patient with abdominal pain or collapse for investigation rather than saying this is a collapse because of $a, b, c$, or $d$. (Excerpt from interview)

This notion of being open to a variety of causes for the patient's clinical presentation, being prepared to accept the general and not have to arrive at a specific diagnosis, represents an element of flexibility and openness that appears to be a further characteristic of the expert practitioner. As discussed earlier, the professional's capacity to consider multiple means and ends is a characteristic of expert judgment making, and this is evidenced through the above behaviour - a reluctance to categorise patients and therefore limit your possibilities. This lends itself with the ability to change the course of management, make amended judgments, based on the acquisition of new evidence or information. Dunne[2] suggests a person of judgment '...does not impose a Procrustean application of the general rule (Procrustes was the character in Greek myth who stretched people or lopped off bits of them to make them fit the bed he had designed for their captivity)'. The expert practitioner would seem to display a form of reflexivity in their approach to patients and the problems they present. This affords the expert practitioner the opportunity to consider multiple means and multiple ends to clinical problems and not be directed by established rules and guidelines.

\section{Making Judgments}

The expert practitioner's ability to make quick, sound judgments and choose whether to act or not act, is hinted at in an earlier excerpt, and is elaborated by other participants - the ability of the expert practitioner to perform several tasks concurrently, perhaps without having all information available to them at the time.

I think you tend to take in a lot more information, that you're not being driven down just a step by step process. The way my head gets around it is I'm trying to take in all different information and not just have it in the consecutive order that I'm going to do certain tasks. (Excerpt from interview)

Despite this ability to absorb a variety of information sources concurrently and direct attention quickly to the problem at hand, the expert practitioner, when faced with difficult or confusing situations, is able to resort to the tried and true basics in order to manage such a situation and make appropriate judgments.

So I suppose each time when something really new comes along you've just got to go back to that step by step approach but you also bring back with you lots of helpful information from your experience. (Excerpt from interview)

Demonstration of the above characteristics was observed during several of the cases attended. During the more routine cases there appeared very little clinical communication between the paramedic crew; however, management was instigated in a timely fashion and seemed to flow smoothly. The more difficult, non-routine cases called for a little more urgency in the patient management. The relaxed mannerisms evident in the routine cases were no longer present, but this did not result in any undue haste or indeed panic but rather an increased receptiveness to stimuli, the capacity for the paramedic to perform tasks concurrently as recognised in earlier excerpts. This tendency to resort to general problem solving heuristics when confronted with unfamiliar problems is similar to strategies adopted by novices; however, the expert has the capacity to operate with greater facility than a novice.[11] This appears to conflict with earlier concepts of the expert's approach to non-routine situations. 
Beckett[12] suggests judgments made in these situations calls for an abandonment of pattern recognition (as there is no pattern applicable to the new situation) and instead the practitioner relies more on anticipative action - a combination of an ability to decide the appropriateness of various courses of action and the confidence to 'try'. This contrasting view is elaborated in the following excerpt:

I think the longer you stay in the job of making these decisions, I think you become more comfortable with having a bit of a dip at something. Probably prepared to back your judgment a bit more and not feel so inclined to think about the negative but more inclined to think about the positive outcomes for the patient. Sometimes you do back your judgment and it doesn't quite work but I figure that as long as you learn from that and you hope that the longer you stay on the less that happens.

Several issues are identified here. The paramedic has recognised the development of confidence in making judgments - the capacity to act anticipatively. However, it is also suggested that this ability is developed through experience and constant feedback from previous actions. The point is also raised how important it is to learn from experiences, be they good or bad. This view is also emphasised by Dunne[2] who states '...raw experience is not a sufficient condition: what is crucial, rather, is that one learns from one's experience perhaps especially from one's mistakes - so that one's experience is constantly reconstructed'.

Interestingly, several of the participants identified an ability to be more accepting of the unknown, be prepared to follow a hunch. The terms 'instinct' and 'gut feeling' were used as an attempt to describe the elusive nature of the making of such decisions.

It's interesting because sometimes you know you're right or moving in the right direction but you still have a bit of a niggling doubt - is this still the right thing. But I think it's the number of things you've seen and you've just put them into a knowledge base that's sitting there, that you seem to hang on to and that seems to get drawn on - but how do you express it, it's very hard to express, very hard to explain. (Excerpt from interview)

Despite attempts to draw out an understanding of why judgments are made and what knowledge base is being drawn on to make these judgments, a lot of these actions still cannot be made explicit by the expert practitioner. However, the reasoning as to why expert practitioners are able to formulate judgments in the field has been shown to relate to a variety of ways of gathering, processing and using the information around them. This developmental step appears to instil in the expert practitioner a confidence to manage the unique and unusual situations. In making judgments they are simultaneously constructing new knowledge and new meaning to presenting problems that enables them to go on. This in turn develops over time through experience obtained within a specific workplace context and inevitably facilitates the growth of both personal and professional knowledge.

\section{Education Initiative}

Several avenues to investigate education and training strategies to assist in the development of tacit knowledge and the ability to perform sound judgments arise from this study. These revolve around developing suitable simulation and scenario facilities that adequately capture the richness and complexity of the workplace context, as well as the diversity and complexity of problematic situations requiring judgment that arise as a component of the paramedic's practice. Secondly, the continual reinforcement of established rules and guidelines, such as the clinical problem solving process, will assist both expert and novice paramedics develop their capacity to make sound clinical judgments in both routine and non-routine situations. Finally, it would be beneficial for both novice and expert paramedics to introduce reflection on their practice as a routine, with formal programs developed should this be considered necessary. New knowledge is constructed as paramedics formulate strategies to make 
judgments when facing unique situations. Therefore avenues need to be made available to assist paramedics to make such learning explicit, through reflection, and incorporate it into their established practices.

\section{Conclusion}

This study has attempted to uncover the nature of professional judgments made by paramedics when they are faced with unique or non-routine situations. The generation of such decision-making has traditionally been associated with the development of tacit knowledge; however, attempts to study the nature of this knowledge source have proved problematic. The term 'tacit knowledge' introduces ambiguity, as it is confused with and used synonymously with such terms as 'intuition', 'sixth sense' and 'gut feeling'. These concepts introduce a mystical quality to tacit knowledge and impede attempts to better understand the phenomenon, consequently serving to hamper research efforts. The relationship between tacit knowledge and the judgments made as part of expert practice affords the opportunity to shift the research focus away from the elusive tacit knowledge and instead direct efforts toward the more observable manifestation of tacit knowledge that is judgment.

The data has uncovered a rich interplay of factors contributing to the development of judgment-making capabilities in expert practitioners. Certainly experience within a particular context is involved; however, this alone is not sufficient. Paramedics identified both the reversion to basics, tried and trusted rules or guidelines, and the capacity to play a hunch and trust your judgment, as also assisting them in making judgments when facing unique situations, even though these features appear incommensurable. Bringing forward a further observation, the capacity to manage multiple sources of information concurrently, can rectify this apparent contradiction. Paramedics, by their own admission, utilised multiple sources of information when making judgments, therefore the capacity exists to examine established heuristics and simultaneously search for alternative strategies.

Paramedics also expressed a reluctance to prematurely allocate patients into specific management protocols, that is, they displayed a willingness to deviate from previously determined management pathways should circumstances alter. It was suggested novice practitioners were less flexible, tending to adhere to specific management protocols once initiated. This trait, the capacity to recognise multiple means to multiple ends, complements the expert practitioner's ability to face uncertainty with confidence and display the 'anticipative action' described by Beckett.[12] Judgment-making involves a constant shifting between established heuristics and previously untried and untested management strategies, driven by a confidence to 'try'. Paramedics are not only relying on existing knowledge, both explicit and tacit, but they are generating new knowledge and understanding as they confront unique situations in the workplace.

This study has directed the way for further research into the manner in which information is managed by both novice and expert practitioners. An understanding of the forms of pattern recognition adopted by novice and expert practitioners may provide pathways to enhance this process. Appreciation of the difference between how novice and expert practitioners approach unique situations, and how they utilise their knowledge sources, may provide insight into how knowledge and meaning is constructed in the workplace and serve to guide future education and training initiatives. 


\section{REFERENCES}

1. Edwards, R. (1997) Changing Places? Flexibility, Lifelong Learning and a Learning Society, Routledge, London.

2. Dunne, J. (1999) Professional Judgement and the Predicaments of Practice. European Journal of Marketing, 33, 707-719.

3. Hager, P. (2000) Know-How and Workplace Practical Judgement. Journal of Philosophy of Education, 34, 281-296.

4. Hackley, C. (1999) Tacit Knowledge and the Epistemology of Expertise in Strategic Marketing Management. European Journal of Marketing, 33, 720-735.

5. Daley, B. (2001) Learning and Professional Practice: a Study of Four Professions. Adult Education Quarterly, 52, 39-48.

6. Beckett, D. and Hager, P. (2000) Making Judgments as the Basis for Workplace Learning: Towards an Epistemology of Practice. International Journal of Lifelong Education, 19, 300-311.

7. Ambrosini, V. and Bowman, C. (2001) Tacit Knowledge: Some Suggestions for Operationalization. Journal of Management Studies, 38, 811-829.

8. Merriam, S. (1998) Qualitative Research and Case Study Applications in Education, Jossey-Bass, San Francisco.

9. Wenger, E. (1998) Communities of Practice: Learning, Meaning, and Identity, Cambridge University Press, Cambridge MA.

10. Epstein, R. (1999) Mindful Practice. JAMA, 282, 833-849.

11. Glaser, R. (1999) In Learning and Knowledge(Eds, McCormick, R. and Paechter, C.) Sage Publications, London.

12. Beckett, D. (2000) Making Workplace Learning Explicit: An Epistemology of Practice for the Whole Person. Westminster Studies in Education, 23, 41-53.

This article was peer reviewed for the Journal of Emergency Primary Health Care Vol. 1 (2-3), 2003 